[8] J. M. C. Clark, "The design of robust approximations to the stochastic differential equations of nonlinear filtering," in Communication Systems and Random Process Theory, J. K. Skwirzynski, Ed. Groningen: Sithoff and Noordhoff, 1978.

[9] M. H. A. Davis, "Pathwise nonlinear filtering," in Stochastic Systems, M. Hazewinkel and J. Willems, Eds. Reidel: Dordrecht, 1981.

[10] A. De Santis, A. Gandolfi, A. Germani, and P. Tardelli, "Polynomial approximation for a class of physical random variables," Proc. AMS, vol. 120, pp. 261-266, 1994.

[11] A. Gandolfi and A. Germani, "On the definition of a topology in Hilbert spaces with applications to the white noise theory," J. Franklin Inst., vol. 316, pp. 435-444, 1983.

[12] L. Gross, "Measurable functions on Hilbert space," Trans. AMS, vol. 105, pp. 372-390, 1962.

[13] _ _Harmonic analysis on Hilbert spaces," Mem. AMS, vol. 46, 1963

[14] G. Kallianpur and R. L. Karandikar, White Noise Theory of Filtering, Prediction and Smoothing. London, U.K.: Gordon and Breach, 1988.

[15] H. H. Kuo; Gaussian Measures in Banach Spaces, Lecture Notes in Math., vol. 463. New York: Springer-Verlag, 1975.

[16] R. Mazumdar and J. H. Seo, "On the innovations problem in a finitely additive white noise approach to nonlinear filtering," Differential and Integral Equations, vol. 1, no. 2, pp. 231-249, 1988.

[17] R. Mazumdar, R. Kannurpatti, and A. Bagchi, "On input/output maps for nonlinear systems via continuity in a locally convex topology," Syst. Contr. Lett., vol. 24, pp. 273-281, 1995.

[18] D. Ocone, "Multiple integral expansions for nonlinear filtering," Stochastics, vol. 10, pp. 1-30, 1983.

[19] P. M. Prenter, "A Weierstrass theorem for real, separable Hilbert spaces," J. Approximation Theory, vol. 3, pp. 341-351, 1970.

[20] O. G. Smolyanov and A. V. Uglanov, "Every Hilbert subspace of a Wiener space has measure zero," Matem. Zametki., vol. 14, no. 3, pp. 369-374, 1973.

[21] E. Wong and B. Hajek, Stochastic Processes in Engineering Systems. New York: Springer-Verlag, 1983

\section{Limiting Zeros of Decouplable MIMO Systems}

Steven R. Weller

Abstract-For sufficiently rapid zero-order hold (ZOH) sampling, it is known that the zeros of single-input/single-output discrete-time systems expressed in the forward shift operator converge to values determined only by the degrees of the finite and infinite zeros of the underlying continuous-time system. In this paper, it is shown how this result can be generalized to multi-input/multi-output (MIMO) systems decouplable by static-state feedback (equivalently, having a diagonal interactor matrix). In the fast sampling limit, the authors show how invariant and infinite zero structure is mapped under $\mathrm{ZOH}$ sampling for discrete-time systems expressed in either the delta (forward difference) or forward shift operators.

Index Terms-Discrete-time systems, poles, zeros.

\section{INTRODUCTION}

For single-input/single-output (SISO) continuous-time systems having relative degree $r$, the corresponding discrete-time system obtained by zero-order hold $(\mathrm{ZOH})$ sampling has relative degree one for all but a finite set of sampling periods. The additional $r-1$

Manuscript received October 9, 1996; revised June 13, 1997 and September 15, 1997. This work was supported by the Australian Research Council.

The author is with the Department of Electrical and Computer Engineering, and the Centre for Integrated Dynamics and Control (CIDAC), University of Newcastle, Callaghan, NSW 2308, Australia.

Publisher Item Identifier S 0018-9286(99)00594-2. discrete-time zeros are an artefact of the sampling process and are called the sampling zeros.

There is a simple and well-known one-to-one correspondence between continuous-time poles $p$ and their discrete-time counterparts under ZOH sampling: $p \leftrightarrow e^{p T}$, where $T$ is the sampling period. For zeros, the situation is much more complicated, and analysis is generally restricted to sufficiently large or small sampling periods [1]-[3]. For SISO discrete-time systems expressed in the forward shift operator $q$, the limiting values of the discrete-time zeros as the sampling period $T$ is decreased to zero, the so-called limiting zeros, have been known for some time [1], [4]. Specifically, all finite zeros are mapped in the fast sampling limit to the point 1 , while the remaining $r-1$ zeros are given by the roots of polynomials whose coefficients are determined by the continuous-time relative degree, but are otherwise independent of the plant dynamics.

For multi-input/multi-output (MIMO) systems, much less is known about the mapping of finite and infinite zeros under $\mathrm{ZOH}$ sampling. Grizzle and Shor [5] have considered the mapping of infinite zero structure under $\mathrm{ZOH}$ sampling, showing that most MIMO discretetime systems obtained by $\mathrm{ZOH}$ sampling have a trivial structure at infinity (namely, all zeros at infinity are of order one) for all but a finite set of sampling periods. Hayakawa et al. [6] have shown that when the difference between the largest and smallest infinite zero orders of the underlying continuous-time system is less than two, the location of the zeros of the corresponding $\mathrm{ZOH}$-sampled system in the limit as $T \rightarrow 0$ depends only on the infinite zero orders and McMillan degree of the continuous-time system. For continuous-time systems not satisfying this assumption, Hayakawa et al. [6] have demonstrated that the limiting zeros of the $\mathrm{ZOH}$-sampled system depend in general on the (real-valued) parameters in the state-space description of the system. In this paper, we show that for a large class of MIMO systems (whose infinite zero structure is restricted, in a sense to be made precise shortly), the results of Åström et al. [1] and Bondarko [4] can be extended more or less analogously to the MIMO case.

Recently, Tesfaye and Tomizuka [7] (see also [8]) used an asymptotic analysis to obtain the limiting zeros of $\mathrm{ZOH}$-sampled discretetime SISO systems expressed in the delta (also Euler, or forward difference) operator $\delta=(q-1) / T$ [9]. While Tesfaye and Tomizuka's quantification of limiting zeros is much less direct than that of [1] or [4], it has the distinct advantage of using a state-space framework. A means of extending the results to multivariable systems is thereby suggested, and this is the approach taken in the present paper. The main result of this paper is Theorem 4.1, which, in essence, says that for a large class of MIMO systems whose infinite zero structure is "simple," the limiting sampling zeros can be computed from knowledge of the minimum relative degree in each row of the continuous-time transfer matrix in question. In this paper, the notion of "simplicity" of the infinite zero structure is taken to mean decouplability by static-state feedback of the underlying continuous-time system, i.e., that the interactor matrix [10] is diagonal. Thus the results of this paper complement the work of Hayakawa et al. [6], in which was presented an alternative sufficient condition for the limiting sampling zeros to be computable from the infinite zero orders using a quite different notion of simplicity of infinite zero structure. The results in this paper also extend the work of Grizzle and Shor [5] by taking into consideration the mapping of finite zeros under fast $\mathrm{ZOH}$ sampling and by explicitly identifying a class of continuous-time MIMO systems (namely, those having diagonal interactor matrices) for which fast sampling always leads to a trivial structure at infinity. 
This paper is organized as follows. In Section II, we briefly review the infinite zero structure of multivariable systems and the Isidori-Byrnes normal form. In Section III, we present the state similarity transformation which brings the delta operator-based model into normal form, from which the (state feedback) control law required to zero the output can be readily calculated. The limiting zeros can then be obtained as the eigenvalues of the internal dynamics when the output is constrained to be identically zero, as shown in Section IV.

\section{Preliminaries}

A function $f(T)=O(T)$ if $\lim _{T \rightarrow 0} f(T) / T=K$, where $0<K<\infty$, and $f(T)=o(T)$ if $\lim _{T \rightarrow 0} f(T) / T=0$. The set of eigenvalues of a matrix $X$ is denoted by $\lambda(X)$. A proper rational matrix $B(s)$ is said to be biproper if $\operatorname{det}\left(\lim _{s \rightarrow \infty} B(s)\right) \neq 0$. A rational matrix $P(s)$ is said to have an invariant zero $\lambda \in C$ if the rank of $P(\lambda)$ is strictly less than the normal rank of $P(s)$.

\section{THE NORMAL FORM}

We consider square time-invariant multivariable systems described by

$$
\Sigma_{c}:\left\{\begin{array}{l}
\dot{x}=A_{c} x+B_{c} u \\
y=C x
\end{array}\right.
$$

where $x \in \boldsymbol{R}^{n}$ is the state, $u, y \in \boldsymbol{R}^{m}$ are the control and output, respectively, and $P(s)=C\left(s I-A_{c}\right)^{-1} B_{c}$ has full normal rank. The system $\Sigma_{c}$ is assumed to be minimal, with

$$
C=\left[C_{1}^{T}, C_{2}^{T}, \cdots, C_{m}^{T}\right]^{T} \in R^{m \times n}
$$

and

$$
B_{c}=\left[B_{c, 1}, B_{c, 2}, \cdots, B_{c, m}\right] \in \boldsymbol{R}^{n \times m} .
$$

One description of the infinite zero structure of $\Sigma_{c}$ is provided by the Smith-McMillan form at infinity [11], [12].

Theorem 2.1: For every $m \times n$ proper rational matrix $P(s)$ of rank $p$, there exist biproper matrices $B_{1}(s)$ and $B_{2}(s)$ and a unique set of integers $n_{1}^{\prime} \geq n_{2}^{\prime} \geq \cdots \geq n_{p}^{\prime} \geq 0$ such that

$$
P(s)=B_{1}(s) \text { block diag }\left[\frac{1}{s^{n_{p}^{\prime}}}, \cdots, \frac{1}{s_{2}^{n_{2}^{\prime}}}, \frac{1}{s^{n_{1}^{\prime}}}, 0\right] B_{2}(s) .
$$

The integers $n_{i}^{\prime}$ are called the infinite zero orders and are one way of extending the notion of scalar relative degree to the multivariable case. In this paper attention is restricted to square systems decouplable by static state feedback (or simply decouplable systems), characterized by nonsingularity of the decoupling matrix

$$
\left[\begin{array}{c}
C_{1} A_{c}^{r_{1}-1} B_{c} \\
C_{2} A_{c}^{r_{2}-1} B_{c} \\
\vdots \\
C_{m} A_{c}^{r_{m}-1} B_{c}
\end{array}\right] \in \boldsymbol{R}^{m \times m}
$$

where $r_{i}$ is the smallest relative degree among the entries of $P_{i}(s)$, the $i$ th row of $P(s)$, i.e., the smallest integer $r_{i}$ such that

$$
\begin{aligned}
\lim _{s \rightarrow \infty} s^{r_{i}} P_{i}(s) & =\lim _{s \rightarrow \infty} s^{r_{i}} C_{i}\left(s I-A_{c}\right)^{-1} B_{c} \\
& =C_{i} A_{c}^{r_{i}-1} B_{c} \neq 0 .
\end{aligned}
$$

From this definition of $r_{i}$, it follows that

$$
C_{i} A_{c}^{k} B_{c, j}=0
$$

for all $1 \leq j \leq m$, all $1 \leq i \leq m$, and all $k<r_{i}-1$. For square decouplable systems, the infinite zero orders $n_{i}^{\prime}$ and the row relative degrees $r_{i}$ are related as follows [12]:

$$
\left\{n_{1}^{\prime}, n_{2}^{\prime}, \cdots n_{m}^{\prime}\right\}=\left\{r_{1}, r_{2}, \cdots, r_{m}\right\}
$$

and the system $\Sigma_{c}$ is said to have $r=r_{1}+r_{2}+\cdots+r_{m}$ zeros at infinity. For the purposes of this paper, the ordering of the $n_{i}^{\prime}$ are of no importance, and (4)-(7) provide a simple means of calculating the infinite zero orders directly from the state-space model (1) without need of explicitly computing the biproper matrices $B_{1}(s)$ and $B_{2}(s)$ in (3).

To transform system (1) into the Byrnes-Isidori normal form [13], the following state similarity transformation is introduced:

$$
\begin{aligned}
{\left[\xi_{c}^{T}, \eta_{c}^{T}\right]^{T} } & =\left[\xi_{c}^{(1)^{T}}, \xi_{c}^{(2)^{T}}, \cdots, \xi_{c}^{(m)^{T}},(H x)^{T}\right]^{T} \\
& =L_{c} x
\end{aligned}
$$

where

$$
\begin{gathered}
\xi_{c}^{(i)}=\left[C_{i}^{T},\left(C_{i} A_{c}\right)^{T}, \cdots,\left(C_{i} A_{c}^{r_{i}-1}\right)^{T}\right]^{T} x, \\
i=1,2, \cdots, m .
\end{gathered}
$$

In (8), the existence of $H \in \boldsymbol{R}^{(n-r) \times n}$ such that $L_{c}$ is nonsingular is guaranteed by [13, Proposition 5.1.2]. Moreover, in the linear system setting, [13, Proposition 5.1.2] also ensures that $H$ can be chosen such that $H B_{c}=0$, and it is hereafter assumed that $H$ satisfies this condition. Note that insofar as the present paper is concerned, the matrix $H$ need not be computed, it need only exist.

In these new coordinates, the states evolve according to (10), as shown at the bottom of the page, where $*$ denotes terms whose values are of no interest, and all remaining entries are zero unless otherwise indicated. This normal form clearly displays the infinite zero structure as a chain of integrators associated with each output, while the eigenvalues of $Q_{\eta} \in \boldsymbol{R}^{(n-r) \times(n-r)}$ are the invariant zeros of (1).

\section{Discrete-Time Systems in Delta Form}

With $q$ the forward shift operator and $T$ the sampling period, the discrete-time $\mathrm{ZOH}$ representation of the continuous-time system (1) can be written in terms of the $\delta$ operator $(\delta \triangleq(q-1) / T)$ as follows:

$$
\Sigma_{\delta}:\left\{\begin{array}{l}
\delta x[k]=A_{\delta} x[k]+B_{\delta} u[k] \\
y[k]=C x[k]
\end{array}\right.
$$

By defining

$$
B_{1}^{*}=\frac{1}{2 !} A_{c}+\frac{T}{3 !} A_{c}^{2}+\cdots+\frac{T^{k-1}}{(k+1) !} A_{c}^{k}+\cdots
$$

the matrices $A_{\delta}$ and $B_{\delta}$ can be expressed as regular perturbations of their continuous-time counterparts

$$
\begin{aligned}
& A_{\delta}=A_{c}+T A_{1}=A_{c}\left(I+T B_{1}^{*}\right) \\
& B_{\delta}=B_{c}+T B_{1}=\left(I+T B_{1}^{*}\right) B_{c}
\end{aligned}
$$

where

$$
\begin{aligned}
& A_{1}=\frac{1}{2 !} A_{c}^{2}+\frac{T}{3 !} A_{c}^{3}+\cdots+\frac{T^{k-2}}{k !} A_{c}^{k}+\cdots \\
& B_{1}=B_{1}^{*} B_{c} .
\end{aligned}
$$

Suppose the state similarity transformation

$$
\begin{aligned}
{\left[\xi^{T}, \eta^{T}\right]^{T} } & =\left[\xi^{(1)^{T}}, \xi^{(2)^{T}}, \cdots, \xi^{(m)^{T}},(H x)^{T}\right]^{T} \\
& =L x
\end{aligned}
$$

where

$$
\begin{aligned}
\xi^{(i)}= & {\left[C_{i}^{T},\left(C_{i} A_{\delta}\right)^{T}, \cdots,\left(C_{i} A_{\delta}^{r_{i}-1}\right)^{T}\right]^{T} x, } \\
& i=1,2, \cdots, m, \\
= & {\left[\xi_{1}^{(i)}, \xi_{2}^{(i)}, \cdots, \xi_{r_{i}}^{(i)}\right]^{T} }
\end{aligned}
$$


is applied to the system (11). The resulting discrete-time state-space model is

$$
\begin{aligned}
\delta\left[\begin{array}{l}
\xi \\
\eta
\end{array}\right] & =L A_{\delta} L^{-1}\left[\begin{array}{l}
\xi \\
\eta
\end{array}\right]+L B_{\delta} u \\
y & =\left[y_{1}, y_{2}, \cdots, y_{m}\right]^{T} \\
y_{i} & =\xi_{1}^{(i)}, \quad i=1,2, \cdots, m
\end{aligned}
$$

where $L A_{\delta} L^{-1}$ is, to within $O(T)$, equal to the system matrix in the normal form (10) of the underlying continuous-time system. Using (13), (14), and (6), it is possible to obtain the following asymptotic approximation to $L B_{\delta}$, in which the leading order term in each row has been retained:

$$
\begin{aligned}
& L B_{\delta}=\left[\begin{array}{c}
C_{1} B_{\delta} \\
C_{1} A_{\delta} B_{\delta} \\
\vdots \\
C_{1} A_{\delta}^{r_{1}-1} B_{\delta} \\
\vdots \\
C_{m} B_{\delta} \\
C_{m} A_{\delta} B_{\delta} \\
\vdots \\
\frac{C_{m} A_{\delta}^{r_{m}-1} B_{\delta}}{H B_{\delta}}
\end{array}\right] \\
& =\left[\begin{array}{c}
C_{1}\left(I+T B_{1}^{*}\right) B_{c} \\
C_{1} A_{c}\left(I+T B_{1}^{*}\right)^{2} B_{c} \\
\vdots \\
\frac{C_{1} A_{c}^{r_{1}-1}\left(I+T B_{1}^{*}\right)^{r_{1}} B_{c}}{\vdots} \\
\frac{C_{m}\left(I+T B_{1}^{*}\right) B_{c}}{C_{m} A_{c}\left(I+T B_{1}^{*}\right)^{2} B_{c}} \\
\vdots \\
\frac{C_{m} A_{c}^{r_{m}-1}\left(I+T B_{1}^{*}\right)^{r_{m}} B_{c}}{H\left(I+T B_{1}^{*}\right) B_{c}}
\end{array}\right]
\end{aligned}
$$

$$
=\left[\begin{array}{c}
T^{r_{1}-1} \alpha_{0}^{(1)}+O\left(T^{r_{1}}\right) \\
T^{r_{1}-2} \alpha_{1}^{(1)}+O\left(T^{r_{1}-1}\right) \\
\vdots \\
T \alpha_{r_{1}-2}^{(1)}+O\left(T^{2}\right) \\
\alpha_{r_{1}-1}^{(1)}+O(T) \\
\hline \vdots \\
T^{r_{m}-1} \alpha_{0}^{(m)}+O\left(T^{\left.r_{m}\right)}\right. \\
T^{r_{m}-2} \alpha_{1}^{(m)}+O\left(T^{r_{m}-1}\right) \\
\vdots \\
T \alpha_{r_{m}-2}^{(m)}+O\left(T^{2}\right) \\
\alpha_{r_{m}-1}^{(m)}+O(T) \\
\frac{T}{2} H A_{c} B_{c}+O\left(T^{2}\right)
\end{array}\right] .
$$

The terms $\alpha_{j}^{(i)} \in \boldsymbol{R}^{1 \times m}$ for $i=1, \cdots, m$, and $j=1,2, \cdots, r_{i}-1$ play a key role in the formula for the asymptotic location of the sampling zeros of (11), as presented in Theorem 4.1. While it is straightforward to show that

$$
\alpha_{0}^{(i)}=\frac{1}{r_{i} !} C_{i} A_{c}^{r_{i}-1} B_{c} \in \boldsymbol{R}^{1 \times m}, \quad i=1,2, \cdots, m
$$

obtaining closed-form expressions for $\alpha_{j}^{(i)}$ for $i=1,2, \cdots, m$, and $j=1,2, \cdots, r_{i}-1$ requires the evaluation of terms in the expansion of integer powers of $I+T B_{1}^{*}$. This is not, in general, a straightforward task, since $B_{1}^{*}$ is itself a power series in $T$. By careful examination of the terms in each row of $L B_{\delta}$ associated with $C_{i} A_{c}^{r_{i}-1} B_{c}$, it is possible to show that for $i=1,2, \cdots, m$

$$
\begin{aligned}
\alpha_{1}^{(i)} & =\left(\sum_{i=1}^{r_{i}-1} \frac{1}{i !\left(r_{i}-i\right) !}\right) C_{i} A_{c}^{r_{i}-1} B_{c} \\
& =\left(2^{r_{i}}-2\right) \frac{1}{r_{i} !} C_{i} A_{c}^{r_{i}-1} B_{c} \\
& =\tilde{\alpha}_{1}^{(i)} \alpha_{0}^{(i)}, \quad \tilde{\alpha}_{1}^{(i)} \triangleq 2^{r_{i}}-2
\end{aligned}
$$

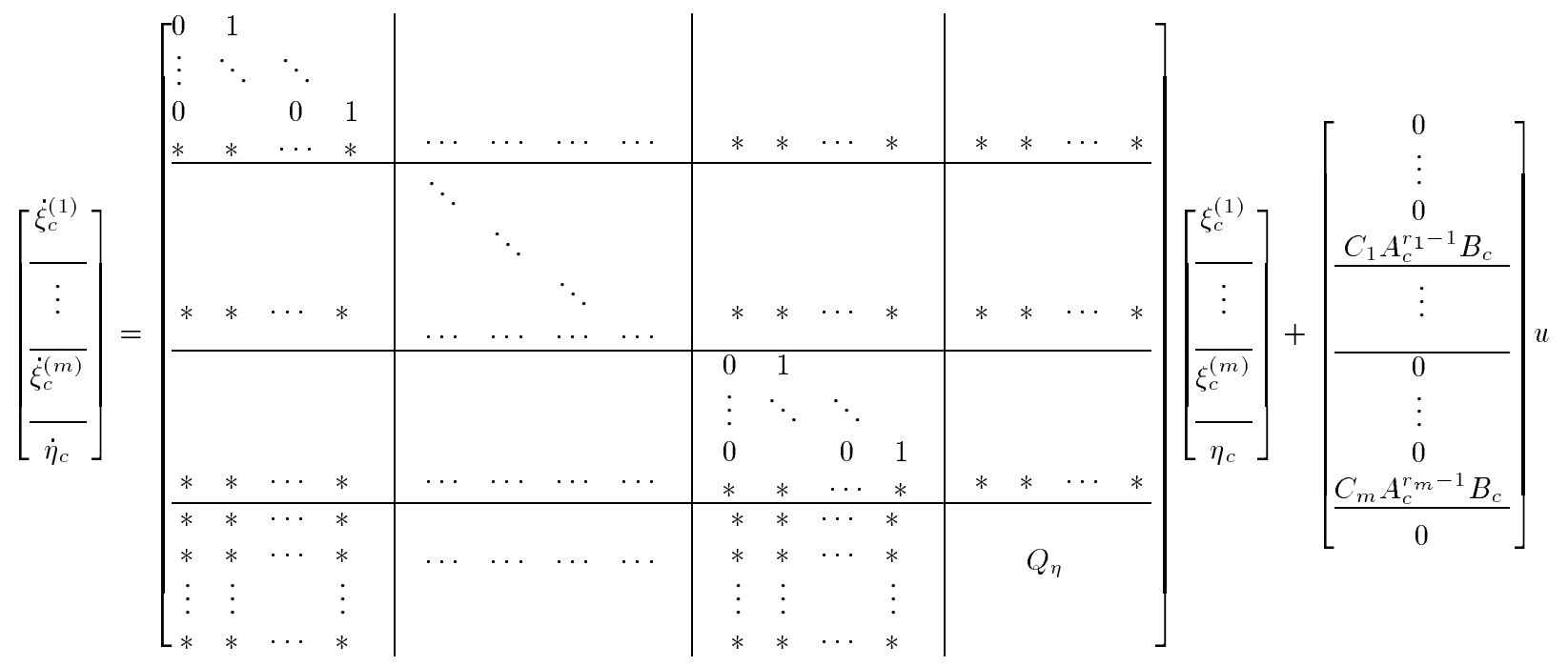




$$
\begin{aligned}
\alpha_{2}^{(i)}= & \left(\sum_{i=1}^{r_{i}-1} \frac{2^{r_{i}-i}-2}{i !\left(r_{i}-i\right) !}\right) C_{i} A_{c}^{r_{i}-1} B_{c} \\
= & \left(3^{r_{i}}-3 \cdot 2^{r_{i}}+3\right) \frac{1}{r_{i} !} C_{i} A_{c}^{r_{i}-1} B_{c} \\
= & \tilde{\alpha}_{2}^{(i)} \alpha_{0}^{(i)}, \quad \tilde{\alpha}_{2}^{(i)} \triangleq 3^{r_{i}}-3 \cdot 2^{r_{i}}+3 \\
\alpha_{3}^{(i)}= & \left(\sum_{i=1}^{r_{i}-1} \frac{3^{r_{i}-i}-3 \cdot 2^{r_{i}-i}+3}{i !\left(r_{i}-i\right) !}\right) C_{i} A_{c}^{r_{i}-1} B_{c} \\
= & \left(4^{r_{i}}-4 \cdot 3^{r_{i}}+6 \cdot 2^{r_{i}}-4\right) \frac{1}{r_{i} !} C_{i} A_{c}^{r_{i}-1} B_{c} \\
= & \tilde{\alpha}_{3}^{(i)} \alpha_{0}^{(i)}, \quad \tilde{\alpha}_{3}^{(i)} \triangleq 4^{r_{i}}-4 \cdot 3^{r_{i}}+6 \cdot 2^{r_{i}}-4 \\
\alpha_{4}^{(i)}= & \left(\sum_{i=1}^{r_{i}-1} \frac{4^{r_{i}-i}-4 \cdot 3^{r_{i}-i}+6 \cdot 2^{r_{i}-i}-4}{i !\left(r_{i}-i\right) !}\right) \\
& \cdot C_{i} A_{c}^{r_{i}-1} B_{c} \\
= & \left(5^{r_{i}}-5 \cdot 4^{r_{i}}+10 \cdot 3^{r_{i}}-10 \cdot 2^{r_{i}}+5\right) \\
& \cdot \frac{1}{r_{i} !} C_{i} A_{c}^{r_{i}-1} B_{c} \\
= & \tilde{\alpha}_{4}^{(i)} \alpha_{0}^{(i)}, \\
\tilde{\alpha}_{4}^{(i)} \triangleq & 5^{r_{i}}-5 \cdot 4^{r_{i}}+10 \cdot 3^{r_{i}}-10 \cdot 2^{r_{i}}+5 .
\end{aligned}
$$

The expressions (22)-(26) are sufficient to deal with continuoustime systems having infinite zero orders $r_{i} \leq 5$ and therefore cover the majority of practical situations. To generate the higher order terms $\alpha_{5}^{(i)}, \alpha_{6}^{(i)}, \cdots$ necessary for dealing with $r_{i} \geq 6$, we can use the binomial theorem to expand the powers of $I+T B_{1}^{*}$ in (20), in conjunction with a recursive procedure for evaluating specified terms in integral powers of power series (see for example [14, p. 14]). Details of such an approach are presented in [8].

\section{ZERO DYNAMICS AND SAMPLING Zeros}

The invariant zeros of linear systems are the eigenvalues of the zero dynamics, the dynamics describing the internal system behavior when the control input and initial conditions are chosen so as to constrain the output to be identically zero [13]. With the delta operator model (19) in Byrnes-Isidori normal form [at least to within $O(T)$ ], the control law which constrains the output to be zero can be readily calculated.

Consider the discrete-time system (19), in which the higher order terms in $L A_{\delta} L^{-1}$ and $L B_{\delta}$ are neglected. Requiring $\delta y_{i}=0$ for all time, and for all $1 \leq i \leq m$, constrains the control input $u$ to satisfy

$$
\delta y_{i}=\delta \xi_{1}^{(i)}=\xi_{2}^{(i)}+T^{r_{i}-1} \alpha_{0}^{(i)} u=0 .
$$

Using matrix notation, the required control law is

$$
u=-\left[\begin{array}{c}
T^{r_{1}-1} \alpha_{0}^{(1)} \\
T^{r_{2}-1} \alpha_{0}^{(2)} \\
\vdots \\
T^{r_{m}-1} \alpha_{0}^{(m)}
\end{array}\right]^{-1}\left[\begin{array}{c}
\xi_{2}^{(1)} \\
\xi_{2}^{(2)} \\
\vdots \\
\xi_{2}^{(m)}
\end{array}\right]=-K^{-1}\left[\begin{array}{c}
\xi_{2}^{(1)} \\
\xi_{2}^{(2)} \\
\vdots \\
\xi_{2}^{(m)}
\end{array}\right]
$$

where for $T>0$ the matrix inverse is guaranteed to exist in view of (22) and the nonsingularity of the decoupling matrix (4). If we define

$$
\bar{\xi}=\left[\bar{\xi}^{(1)^{T}}, \bar{\xi}^{(2)^{T}}, \cdots, \bar{\xi}^{(m)^{T}}\right]^{T}
$$

where

$$
\bar{\xi}^{(i)}=\left[\xi_{2}^{(i)}, \xi_{3}^{(i)}, \cdots, \xi_{r_{i}}^{(i)}\right]^{T}, \quad i=1,2, \cdots, m
$$

then the subspace

$$
\begin{aligned}
\mathcal{V} & =\{x: y=C x=0\} \\
& =\left\{\left(0, \bar{\xi}^{(1)}, 0, \bar{\xi}^{(2)}, \cdots, 0, \bar{\xi}^{(m)}, \eta\right): \bar{\xi}^{(i)} \in R^{r_{i}-1}, \eta \in R^{n-m}\right\}
\end{aligned}
$$

is referred to as the zero dynamics subspace of (19). That is, the dynamics of the $(\bar{\xi}, \eta)$ variables correspond to the internal dynamics of the system when the control input $u$ and initial conditions $\bar{\xi}(0), \eta(0)$ have been chosen in such a way as to constrain the output $y$ to be identically zero for all time.

The following theorem is the main result of the paper and establishes that the high-gain form of the control law (28) as $T \rightarrow 0$ leads to a separation of time scales in the zero dynamics of (and therefore in the limiting zeros of) the discrete-time system $\Sigma_{\delta}$. Moreover, that part of the zero dynamics associated with the fast time scale corresponds to the sampling zeros, while the slow time scale dynamics are associated with the mapping of invariant zeros under $\mathrm{ZOH}$ sampling.

Theorem 4.1: Consider a square, decouplable continuous-time system of the form (1) and its $\mathrm{ZOH}$ equivalent representation (11) expressed in the delta operator. Then if the system (1) has infinite zero orders $r_{1}, r_{2}, \cdots, r_{m}$ with $r=r_{1}+\cdots+r_{m}$, system (11) has $n-m$ invariant zeros which, according to their asymptotic behavior as $T \rightarrow 0$, belong to two groups.

1) The $r-m$ sampling zeros tend asymptotically to the set

$$
\bigcup_{i=1}^{m} T^{-1} \lambda\left(W_{i}\right)
$$

where

$$
W_{i}=\left[\begin{array}{ccccc}
-\tilde{\alpha}_{1}^{(i)} & 1 & 0 & \cdots & 0 \\
-\tilde{\alpha}_{2}^{(i)} & 0 & 1 & & 0 \\
\vdots & & & \ddots & \\
-\tilde{\alpha}_{r_{i}-2}^{(i)} & & & & 1 \\
-\tilde{\alpha}_{r_{i}-1}^{(i)} & 0 & \cdots & \cdots & 0
\end{array}\right] \in \boldsymbol{R}^{\left(r_{i}-1\right) \times\left(r_{i}-1\right)},
$$

2) The remaining $n-r$ invariant zeros tend to the invariant zeros of the continuous-time system (1).

Proof: We begin by noting that the control law (28) assumes each $\xi_{2}^{(i)}$ exists. If for any $1 \leq i \leq m$ it happens that $r_{i}=1$, then the corresponding constraints in (27) are replaced by $y_{i}=\xi_{1}^{(i)}=0$, and the associated $\bar{\xi}^{(i)}$ terms in (32) are nonexistent. Thus if $r_{i}=1$, the corresponding $W_{i}$ matrix is null, implying that no sampling zeros are introduced by infinite zeros of order one, analogous to the scalar case. If (1) has one or more infinite zeros of order one, the state feedback law which zeros the output is no longer unique. For notational simplicity, however, we will assume for the purposes of this proof that control law (28) is used, since it ensures an identically zero output when any finite values are substituted for the corresponding $\xi_{2}^{(i)}$ terms.

From (21)

$$
L B_{\delta} u=-L B_{\delta} K^{-1}\left[\begin{array}{c}
\xi_{2}^{(1)} \\
\xi_{2}^{(2)} \\
\vdots \\
\xi_{2}^{(m)}
\end{array}\right]
$$


where the $i$ th column of $L B_{\delta} K^{-1}$ is given by

$$
\begin{gathered}
{[\underbrace{0,0, \cdots, 0,}_{r_{1}+\cdots+r_{i-1} \text { terms }} 1, \frac{\tilde{\alpha}_{1}^{(i)}}{T}, \frac{\tilde{\alpha}_{2}^{(i)}}{T^{2}}, \cdots, \frac{\tilde{\alpha}_{r_{i}-1}^{(i)}}{T^{r_{i}-1}},} \\
\underbrace{0,0, \cdots, 0,}_{r_{i+1}+\cdots+r_{m} \text { terms }} \underbrace{*, *, \cdots, *}_{n-m \text { terms }}]^{T} .
\end{gathered}
$$

Since $K^{-1}=\bar{K}^{T} / \operatorname{det} K$, where the elements $\bar{k}_{i, j}$ of the matrix $\bar{K}$ are the cofactors of $K$, the entry in row $r_{1}+\cdots+r_{i-1}+1$ and column $i$ of $L B_{\delta} K^{-1}$ is given by

$$
\frac{1}{\operatorname{det} K}\left[T^{r_{i}-1} \alpha_{0,1}^{(i)} \bar{k}_{i, 1}+T^{r_{i}-1} \alpha_{0,2}^{(i)} \bar{k}_{i, 2}+\cdots+T^{r_{i}-1} \alpha_{0, m}^{(i)} \bar{k}_{i, m}\right]
$$

This expression is identically equal to one, however, since the bracketed sum is simply the cofactor expansion of det $K$ along the $i$ th row of $K$. In view of (21) and the relationship between $\alpha_{0}^{(i)}$ and $\alpha_{j}^{(i)}$ for $j \geq 1$ [see (23)-(26)], the subsequent $r_{i}-$ 1 terms in the $i$ th column of $L B_{\delta} K^{-1}$ are therefore given by $\left(\tilde{\alpha}_{1}^{(i)} / T\right),\left(\tilde{\alpha}_{2}^{(i)} / T^{2}\right), \cdots,\left(\tilde{\alpha}_{r_{i}-1}^{(i)} / T^{r_{i}-1}\right)$. Next, consider the entry in the first row and $i$ th column of $L B_{\delta} K^{-1}$

$$
\frac{1}{\operatorname{det} K}\left[T^{r_{1}-1} \alpha_{0,1}^{(1)} \bar{k}_{i, 1}+T^{r_{1}-1} \alpha_{0,2}^{(1)} \bar{k}_{i, 2}+\cdots+T^{r_{1}-1} \alpha_{0, m}^{(1)} \bar{k}_{i, m}\right] .
$$

For $i \neq 1$, this expression evaluates to zero, since the entries $T^{r_{1}-1} \alpha_{0, j}^{(1)}$ and cofactors $\bar{k}_{i, j}$ come from different rows of $K$; the expression (38) therefore equals the determinant of a matrix having two identical rows. It follows then that entries $2,3, \cdots, r_{1}-1$ (and indeed entries $1,2, \cdots, r_{1}+\cdots+r_{i-1}$, together with entries $\left.r_{i}+1, \cdots, r_{1}+\cdots+r_{m}\right)$ of the $i$ th column of $L B_{\delta} K^{-1}$ are also zero, thereby establishing (36).

In view of (19), (35), and (36), it follows that on the subspace $\mathcal{V}$, the dynamics of the $(\bar{\xi}, \eta)$ variables under the state feedback law (28) are given by (39), as shown at the bottom of the page. Following the same line of reasoning as in [7], we cast the dynamics of the $(\bar{\xi}, \eta)$ variables on the subspace $\mathcal{V}$ into a standard singular perturbation form. This can be accomplished by rescaling the $(\bar{\xi}, \eta)$ variables in (39) as follows:

$$
\left[\begin{array}{l}
z \\
\eta
\end{array}\right]=\text { block diag }\left[M_{1}, M_{2}, \cdots, M_{m}, I_{(n-m) \times(n-m)}\right]\left[\begin{array}{l}
\xi \\
\eta
\end{array}\right]
$$

where $M_{i}=\operatorname{diag}\left[1, T, T^{2}, \cdots, T^{r_{i}-2}\right]$. The zero dynamics therefore satisfy

$$
\delta\left[\begin{array}{c}
T z \\
\eta
\end{array}\right]=\operatorname{block} \operatorname{diag}\left[W_{1}, \cdots, W_{m}\right]\left[\begin{array}{c}
z \\
\eta
\end{array}\right]+o(T)
$$

where $W_{i}$ is given by (34) and $Q_{\eta}$ is the matrix whose eigenvalues are the invariant zeros of the continuous-time system (1). Equation (40) is in the standard two time-scale form [15], and since the eigenvalues of the matrix on the right-hand side are asymptotically given by $\lambda\left(W_{1}\right) \cup \cdots \cup \lambda\left(W_{m}\right) \cup \lambda\left(Q_{\eta}\right)$, the dynamics of the $(\bar{\xi}, \eta)$ variables on the subspace $\mathcal{V}$ are those of block $\operatorname{diag}\left[T^{-1} W_{1}, \cdots, T^{-1} W_{m}, Q_{\eta}\right]$ and the result follows.

Equations (33) and (34) are identical in form to the corresponding expressions for the sampling zeros of SISO systems expressed in the delta operator [8]. Since the analogous expressions for shift operatorbased models are well known (see, for example [1] and [4]), we therefore have the following corollary, which pertains to the limiting zeros of the discrete-time system

$$
\Sigma_{q}:\left\{\begin{array}{l}
q x[k]=A_{q} x[k]+B_{q} u[k] \\
y[k]=C x[k]
\end{array}\right.
$$

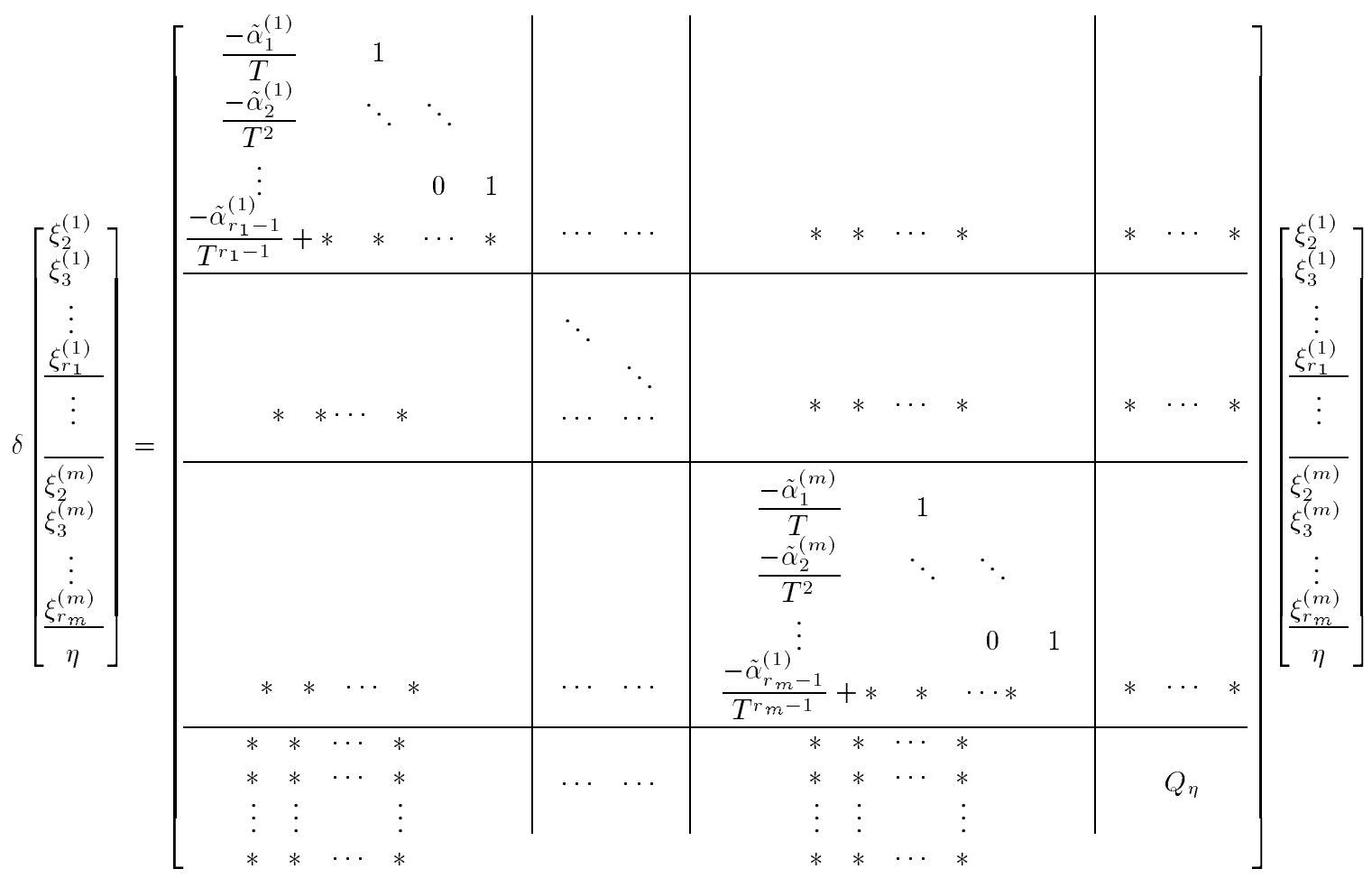


obtained by ZOH sampling of the continuous-time system $\Sigma_{c}$, so that $A_{q}=e^{A_{c} T}$ and $B_{q}=\int_{0}^{T} e^{A_{c}(T-\tau)} B_{c} d \tau$.

Corollary 4.1: Consider a square, decouplable continuous-time system of the form (1) and its $\mathrm{ZOH}$ equivalent representation (41) expressed in the shift operator. Then if system (1) has infinite zero orders $r_{1}, r_{2}, \cdots, r_{m}$ with $r=r_{1}+\cdots+r_{m}$, system (41) has $n-m$ invariant zeros which, according to their asymptotic behavior as $T \rightarrow 0$, belong to two groups.

1) The $r-m$ sampling zeros tend to the roots of the reciprocal polynomials

$$
\begin{gathered}
B^{(i)}(z)=b_{r_{i} 1} z^{r_{i}-1}+b_{r_{i} 2} z^{r_{i}-2}+\cdots+b_{r_{i} r_{i}} \\
i=1,2, \cdots, m \\
b_{r_{i} j}=\sum_{k=1}^{j}(-1)^{j-k} k^{r_{i}}\left(\begin{array}{c}
r_{i}+1 \\
j-k
\end{array}\right), \quad j=1,2, \cdots, r_{i} .
\end{gathered}
$$

2) The remaining $n-r$ invariant zeros approach the point $z=1$.

\section{CONCLUSIONS}

In this paper, we have shown that for continuous-time MIMO systems having a diagonal interactor matrix, the zeros of the corresponding $\mathrm{ZOH}$-sampled system (in the fast sampling limit) depend only on the infinite zero orders and invariant zeros of the underlying continuous-time system. Theorem 4.1 and Corollary 4.1 provide the means for calculating these limiting zeros for discrete-time systems expressed, respectively, in the delta and forward shift operators. Further research is needed to establish connections between the results in this paper and those of Hayakawa et al. [6], wherein a quite distinct notion of simplicity of infinite zero structure is used.

\section{REFERENCES}

[1] K. J. Åström, P. Hagander, and J. Sternby, "Zeros of sampled systems," Automatica, vol. 20, no. 1, pp. 21-38, 1984.

[2] T. Hagiwara, T. Yuasa, and M. Araki, "Stability of the limiting zeros of sampled-data systems with zero- and first-order holds," Int. J. Contr., vol. 58 , no. 6 , pp. $1325-1345,1993$.

[3] T. Hagiwara, "Analytical study on the intrinsic zeros of sampled-data systems," IEEE Trans. Automat. Contr., vol. 41, pp. 261-263, Feb. 1996.

[4] V. A. Bondarko, "Discretization of continuous linear dynamic systems-Analysis of the methods," Syst. Contr. Lett., vol. 5, pp. 97-101, Nov. 1984.

[5] J. W. Grizzle and M. H. Shor, "Sampling, infinite zeros and decoupling of linear systems," Automatica, vol. 24, no. 3, pp. 387-396, 1988.

[6] Y. Hayakawa, S. Hosoe, and M. Ito, "On the limiting zeros of sampled multivariable systems," Syst. Contr. Lett., vol. 2, no. 5, pp. 292-300, Feb. 1983.

[7] A. Tesfaye and M. Tomizuka, "Zeros of discretized continuous systems expressed in the Euler operator-An asymptotic analysis," IEEE Trans. Automat. Contr., vol. 40, pp. 743-747, Apr. 1995.

[8] S. R. Weller, "Comments on 'Zeros of discretized continuous systems expressed in the Euler operator-An asymptotic analysis,' "IEEE Trans. Automat. Contr., vol. 43, pp. 1308-1310, Sept. 1998.

[9] R. H. Middleton and G. C. Goodwin, Digital Control and Estimation: A Unified Approach. Englewood Cliffs, NJ: Prentice-Hall, 1990.

[10] W. A. Wolovich and P. L. Falb, "Invariants and canonical forms under dynamic compensations," SIAM J. Contr. Optimization, vol. 14, no. 6, pp. 996-1008, Nov. 1976

[11] G. Verghese, "Infinite-Frequency behavior in generalized dynamical systems," Ph.D. dissertation, Dept. of Electrical Engineering, Stanford Univ., Stanford, CA, Dec. 1978.

[12] J. F. Lafay, P. Zagalak, and A. Herrera H., "Reduced form of the interactor matrix," IEEE Trans. Automat. Contr., vol. 37, pp. 1778-1782, Nov. 1992.
[13] A. Isidori, Nonlinear Control Systems, 3rd ed. Berlin, Germany: Springer-Verlag, 1995.

[14] I. S. Gradshteyn and I. M. Ryzhik, Table of Integrals, Series, and Products. San Diego, CA: Academic, 1980.

[15] P. Kokotović, H. Khalil, and J. O'Reilly, Singular Perturbations in Control: Analysis and Design. New York: Academic, 1986.

\section{Discrete-Time Modeling of Sampled-Data Control Systems with Direct Feedthrough}

\author{
Marian J. Blachuta
}

Abstract-Discrete-time models of sampled-data control systems are addressed when both a continuous-time plant and a discrete-time controller have a feedthrough. It is pointed out that in this case discrete-time models which can be found in most references should not be used in the closed-loop context. A new state-space model appropriate for the closedloop modeling and formulas for calculating the related discrete-time pulse transfer functions are derived. Intersample phenomena are studied and the feasibility of that model to describe systems with parasiting dynamics is emphasized. Examples from the literature illustrate the relevance of the issue.

Index Terms - Causality, discretization, sampled-data control, sampling.

\section{INTRODUCTION}

In the paper, a model of a classical sampled-data control system as presented in Fig. 1 is considered. It consists of a single-input/singleoutput linear continuous-time plant, a zero-order hold, two switches, and a discrete-time control algorithm. A sampling instant at which the reading of the output is performed is denoted $t_{i}^{s}$, and $t_{i}^{m}$ is a modulation instant at which the control signal changes its value. The normal situation, depicted in Fig. 1, is when sampling takes place prior to modulation $t_{i}^{s}<t_{i}^{m}$. In the paper synchronous sampling is considered, where $t_{i}^{m} \rightarrow t_{i}^{s} \rightarrow t_{i}=i h$. This means that the processing time $\tau$ (necessary for $A / D$ conversion, computation of control and $D / A$ conversion), which can be modeled by a delayed action of the second switch, is assumed to be negligible compared with the sampling period $h$. Practical instrumentation of this scheme involving elements of data conversion hardware, and microprocessor implementation of digital control strategies, can be found in many textbooks, e.g., [6], [9], [11], and [22].

In [13] and [15] models of sampled systems are introduced which, in contrast to the situation depicted in Fig. 1, are based on the reversed order of events where updating of control precedes output reading. This is in discrepancy with practical solutions and can lead to serious problems discussed further.

A general case when the model of a system to be controlled is allowed to have a direct coupling between input and output is addressed. Such a system can be seen as a model in which some parasitic dynamics have been neglected for simplification. If there is

Manuscript received May 4, 1995; revised March 29, 1996. This work was supported by the Polish Committee for Scientific Research (KBN) under Grant 8T $11 \mathrm{~A} 03110$.

The author is with the Silesian Technical University, Department of Automatic Control, PL 44-101, Gliwice, Poland.

Publisher Item Identifier S 0018-9286(99)00595-4. 